\title{
Detection and Localization of Terrestrial L Band RFI with GNSS Receivers
}

This paper was downloaded from TechRxiv (https://www.techrxiv.org).

\section{LICENSE}

CC BY 4.0

SUBMISSION DATE / POSTED DATE

$11-06-2021 / 12-06-2021$

\section{CITATION}

Roberts, Max; Meehan, Thomas K.; Straus, Paul R.; Tien, Jeffery Y.; Valant-Weiss, Bonnie L.; Yizengaw, Endawoke; et al. (2021): Detection and Localization of Terrestrial L Band RFI with GNSS Receivers.

TechRxiv. Preprint. https://doi.org/10.36227/techrxiv.14772900.v1

$\mathrm{DOI}$

10.36227/techrxiv.14772900.v1 


\title{
Detection and Localization of Terrestrial L-Band RFI with GNSS Receivers
}

\author{
T. Maximillian Roberts, Thomas K. Meehan, Paul R. Straus, Jeffery Y. Tien, Bonnie L. Valant-Weiss, \\ Endawoke Yizengaw, and Lawrence E. Young
}

\begin{abstract}
GNSS signals are critically important for a wide range of commercial, military, and science applications. Recent studies have identified threats to the performance of GNSS from both intended and unintended sources of radio frequency interference (RFI). Understanding the distribution of the sources of RFI and the nature of the signals they are emitting is critical to determine and mitigate their effects on the measurements made by GNSS receivers. Terrestrial RFI can be substantially detrimental to the received GNSS signals, affecting the interpretation of related science measurements. NASA's Blackjack/TriG GNSS receivers are used for precise-orbit determination and radio occultation measurements, providing a data record spanning most of the Earth's surface for nearly 20 years. We have developed a highly sensitive detection algorithm which uses variations in the measured signal to noise ratio (SNR), on the order of 10-50 seconds, common to all satellites to identify times and locations subject to RFI. Initial work has focused primarily on detection of the presence of RFI and using the receiver's orbital solution to record the location of detection events. Our inter-mission analysis creates a unique record of global RFI with the potential for a) rigorous detection of the presence of interfering signals during science measurements, b) geolocation of RFI sources, and c) characterization of the nature of the transmitted signal to better identify intent. Preliminary analysis has shown the presence of RFI is well correlated with regional conflicts and other geopolitical activity.
\end{abstract}

Index Terms-Radio frequency interference, RFI, GNSS, GNSS-RO, ionosphere, scintillation, jamming, spoofing

\section{INTRODUCTION}

Global Navigation Satellite Systems (GNSS), such as the Global Positioning System (GPS), are a critical global infrastructure for a range of military, commercial, and science applications that affect almost every part of daily life. The GPS satellite constellations (about 28 satellites) orbit at 20,200 $\mathrm{km}$ altitude, below geostationary orbit but far above most low earth orbiting (LEO) satellites. GNSS satellites transmit highly

T. M. Roberts is with the Jet Propulsion Laboratory, California Institute of Technology, Pasadena, CA, USA. e-mail: t.maximillian.roberts@jpl.nasa.gov.

T. K. Meehan is with the Jet Propulsion Laboratory, California Institute of Technology, Pasadena, CA, USA. e-mail: thomas.k.meehan@jpl.nasa.gov.

P. R. Straus is with the Space Science Application Laboratory, The Aerospace Corporation, El Segundo, CA, USA. e-mail: paul.r.straus@aero.org.

J. Y. Tien is with the Jet Propulsion Laboratory, California Institute of Technology, Pasadena, CA, USA. e-mail: jeffrey.y.tien@jpl.nasa.gov.

B. L. Valant-Weiss is with the Weather Remote Sensing Systems Office, The Aerospace Corporation, El Segundo, CA, USA. e-mail: bonnie.l.valantweiss@aero.org.

E. Yizengaw is with the Space Science Application Laboratory, The Aerospace Corporation, El Segundo, CA, USA. e-mail: endawoke.yizengaw@aero.org.

L. E. Young is with the Jet Propulsion Laboratory, California Institute of Technology, Pasadena, CA, USA. e-mail: lawrence.e.young@jpl.nasa.gov. structured signals which carry information on the location of the transmitters, allowing users (receivers on the ground and on board LEO satellites) to determine their position (and time) - up to centimeter accuracy [1], [2]. Due to the distance of the GNSS satellites, the signal power is substantially reduced at the surface of the Earth, well below the noise floor at the carrier frequency, but is detectable through a matched filtering process.

Government, industry, and scientists have become increasingly dependent on these weak signals to provide position, navigation, and timing (PNT) for other fundamental infrastructure including national security systems, timing of vital computer networks, and everyday civilian navigation. A catastrophic breakdown of any one of these systems could occur via GPS outage [3]. While natural sources of interference such as solar flares [4] and ionospheric density irregularity [5] possess the capability to interfere with signals on a global scale, more localized disturbances are also a problem. Recent studies have identified potential threats to the performance of GNSS from both intended [6], [7] and unintended [8] sources of radio frequency interference (RFI). GNSS jammers are often implemented for military purposes by governments and independent groups to deny regional access to GNSS capabilities, and more recently for preventing the use of unmanned aerial vehicles (drones) [9]. The ground-based implications of these sources may be limited to the local vicinity, but their effects on the measurements made by many orbital GNSS receivers can range hundreds of kilometers [10]-[12]. RFI sources have also been observed to interfere with Automatic Dependant Surveillance Broadcast (ADS-B) transponder systems on aircraft, posing an immediate safetyof-life concern [13].

Understanding the distribution of the sources of RFI and the nature of the signals they are transmitting is critical to determine and mitigate their impact on GNSS-based measurements. Most low Earth orbit (LEO) satellites orbit the planet with GNSS receivers on board to determine their location and to characterize the Earth's atmosphere and surface, leveraging the GNSS signals as scientific probes. Atmospheric and climate scientists are becoming increasingly dependent on GNSS signals for certain observations. For example, it is likely that global soil moisture estimation will be reliant on GNSS surface reflections once the Soil Moisture Active Passive (SMAP) mission [14] concludes its operations as no replacement is yet being developed. RFI sources will perturb or prevent measurements such as these, potentially limiting our understanding of the changing climate. In addition, knowledge 


\begin{tabular}{|c|c|c|c|c|}
\hline $\begin{array}{l}\text { TriG/Blackjack } \\
\text { Receiver Mission } \\
\text { Characteristics }\end{array}$ & & & & 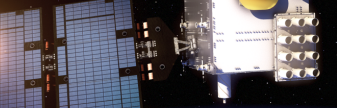 \\
\hline Mission & GRACE & cosmic & GRACE-FO & COSMIC-2 \\
\hline Launch - Termination & March 2002 - November 2017 & April 2006 - September 2018 & May 2018 - Operational & June 2019 - Operational \\
\hline Altitude / Inclination & $500 \mathrm{~km} / 89^{\circ}$ & $800 \mathrm{~km} / 72^{\circ}$ & $500 \mathrm{~km} / 89^{\circ}$ & $540 \mathrm{~km} / 24^{\circ}$ \\
\hline Antenna Boresight & Zenith & FWD/ AFT $15^{\circ}$ above horizon & Zenith & FWD/ AFT $15^{\circ}$ above horizon \\
\hline Front End Filter & $20 \mathrm{MHz}$ at L1 and L2 & $20 \mathrm{MHz}$ at L1 and L2 & $150 \mathrm{MHz}$ at $\mathrm{L} 1$ and $\mathrm{L} 2$ & $150 \mathrm{MHz}$ at $\mathrm{L} 1$ and $\mathrm{L} 2$ \\
\hline Number of Satellites & 2 & 6 & 2 & 6 \\
\hline RFI Sensitivity & Lowest & High & Low & Highest \\
\hline
\end{tabular}

Fig. 1. Timelines and parameters of the four missions discussed in this work, displaying the near 20 year record of global coverage. Qualitative statement of RFI sensitivity relative to other missions in table is a function of the receiver antenna design and orientation, as well as filtering and spacecraft altitude.

of the RFI environment and location of interference sources is useful to understand the impact on systems that depend on GNSS signals.

In this manuscript we will discuss an analysis technique where data from GNSS receivers onboard LEO satellites are used to detect the presence of RFI incident on the spacecraft antenna. While this work is preliminary, the method enables us to estimate the location of RFI transmitters and demonstrates the potential for characterization of the RFI. Section II presents a brief overview of GNSS-based science measurements, the receivers used by an array of NASA missions, and reviews a few forms of terrestrial RFI. Section III details the impact of RFI on the signals tracked by our receivers, and the algorithm developed to detect RFI. Section IV explores the output of this algorithm with 20 years of GNSS data using a variety of techniques to visualize and interpret the nature and location of the RFI sources, then concludes with some discussion of implications and future work.

\section{BACKGROUND}

\section{A. Science Applications of GNSS}

GNSS transmitters produce well defined and predictable signals, which have been proven useful as probes of the Earth's atmosphere and surface. These "signals of opportunity" provide a low cost mechanism to make global observations with high spatial and temporal resolution. Measurement of the direct signal from orbital and ground based receivers can provide estimates of total electron content (TEC) of the ionosphere by comparing the time delay and phase advance difference between the two transmitted frequencies [15]. From an orbital platform, measurement of signals that traverse through the neutral atmosphere can provide information on atmospheric temperature, pressure, and precipitation. This method, commonly referred to as GNSS radio occultation (GNSS-RO) [16] has become the basis of several missions, including COSMIC [17] and COSMIC-2 [18]. Similarly, by looking at GNSS signals reflected from the surface of the planet from an orbital receiver (GNSS-R), estimation of ocean wind speed, surface height, and soil moisture [19]-[21] have been performed, and these measurements form the basis of the CYGNSS [22] missions.

The above examples demonstrate a wide range of applications of GNSS signals which only tangentially leverage a GNSS system's fundamental capability, i.e. accurate receiver localization, or, for the case of a satellite, precise orbit determination (POD). Localization applications for personal, commercial, and military purposes are abundant, but have also proven invaluable for a variety of science observations. Measurements from an array of stable ground stations [23] have provided accurate measurement of plate tectonics with a previously inaccessible accuracy and coverage, and similarly enabled observation and localization of seismic activity with high resolution [24]-[27]. Missions like GRACE [28] and GRACE-FO [29] are able to measure slight variations in Earth's gravitational field, making accessible observation of variations in ground water content, a vital component in understanding the Earth's hydrological cycle [30]-[32].

All of these important measurements of our Earth and its environment are based on the assumption that the receiver is collecting signals with characteristics matching the specifications of the GNSS transmitters. When these signal parameters change, the dependent scientific measurements are perturbed. The spatially and temporary varying effects of intentional and unintentional RFI also produce errors, and need to be quantified to better inform the users of these GNSS-based datasets.

\section{B. TriG/Blackjack GNSS Receivers}

Several generations of NASA's primary GNSS receiver have been developed at JPL and flown on numerous missions, four of which are detailed in the table of Figure 1. These instruments are based on the same fundamental principles implemented in the original Rogue receiver developed in the 1980s, and subsequently adapted into a flight receiver known 
as the TurboRogue Space Receiver (TRSR) [33], designed primarily for precise positioning of the receiving spacecraft, which flew on missions including GPSMet, Oersted, and Sunsat.

The GRACE and GRACE-FO (GFO) missions implement a subsequent generation of receiver referred to as the Blackjack. These missions are critically dependent on the POD capabilities of the receiver, achieving position determined to the centimeter level, and sub-nanosecond time transfer, to enable precise microwave ranging between the paired spacecraft. The receiver was additionally modified to perform radio occultation (RO) measurements. GRACE-FO, an operational follow-on misson to GRACE, has a similar form but implemented changes to the receiver's filtering and antenna design. The BlackJack receiver's RO capability was used as the primary measurement of the first COSMIC radio occultation constellation of 6 satellites. This 12 year mission produced RO measurements across a substantial fraction of the Earth's surface and demonstrated the feasibility of GNSS-RO for numerical weather processing. This receiver generation was flown on over a dozen other missions, including the GRAIL lunar satellites.

The state-of-the-art form of this receiver is known as the TriG, and is a critical instrument on several current and upcoming missions including COSMIC-2, Sentinel-6, Surface Water and Ocean Topography (SWOT), and NASA-ISRO Synthetic Aperture Radar (NISAR). On recently launched COSMIC2, six satellites each carry a TriG receiver capable of POD and GNSS-RO for ionosphere and neutral atmosphere studies. Similar to the first COSMIC mission, these spacecraft feature high-gain actively steered antennas on the ram and anti-ram direction of the spacecraft, yielding increased directionality to the measurements that will play a role in RFI sensitivity. COSMIC-2, like GRACE-FO, also changed antenna designs to a choke-ring antenna from the earlier COSMIC mission's patch-style antenna.

\section{Disrupting GNSS Receivers}

GNSS signal reception can be affected by a number of sources, both intentional and unintentional. Unintentional sources include effects from space weather and other extraterrestrial events, as well as several military, commercial, and civilian sources. Distance measuring equipment (DME) and tactical air navigation (TACAN) used with certain aeronautical navigation systems occupy the same frequency band as the GPS L5 signal [34]. Several developing telecommunication networks [35] operate adjacent to GNSS bands, also potentially interfere with with GNSS receivers. Understanding the impact of these systems on GNSS-based science and PNT in general is an active area of research.

Intentional sources of L-band RFI come in two primary types; jammers and spoofers. Jammers are designed to locally restrict a receiver from tracking GNSS signals by emitting noise in an overlapping band. The noise can be narrowband, targeted to the carrier frequency of a certain signal, or spread across a wide spectrum. An effective form of jamming attempts to match the spread spectrum produced by the GPS chipping codes. All of these techniques act to prevent local receivers from easily locking onto GNSS signals, or, with a bit more power and/or signal complexity, will cause signals being tracked to be dropped.

Spoofers employ a further level of sophistication, designed explicitly to confuse a local receiver. These transmitters emit a signal identical to the GNSS signals that the receivers are trying to find, including data bits. These data can be manipulated to corrupt a receiver's calculations or even trick the receiver into incorrect location determination. In a simple case, this false signal will cause the receiver to track invalid transmitters, which wastes on-board resources. In the worst case, the signal carefully misleads the receiver in a manner that leads the vehicle astray by causing it to perceive it's location incorrectly [36]. An example of this is drone capture activity of Iran, which has been attributed to exploiting a GPS-base weakness of the RQ-170 [9]. A more recent incident involved a US Navy Patrol boat accidentally straying into Iranian waters [7].

\section{Detection OF RFI With $\mathrm{SNR}_{\mathrm{V}}$}

The Blackjack/TriG receivers report the voltage signal-tonoise ratio, $\mathrm{SNR}_{\mathrm{V}}$ [37]. This parameter is affected by a number of factors including the transmitter power, receiving antenna gain patterns, as well as system noise contributions internal and external to the receiver. Figure 2 illustrates conditions of various signal paths between transmitters and a receiver, where the pink regions represent a type of ionospheric plasma variation or irregularity that can cause RF scintillation. Scintillation manifests itself as random variation in the received signal strength from constructive/destructive interference of the signal passing through the plasma irregularity region. Different transmitter orientations will result in signals cutting different chords through the ionosphere, and as the variations along these chords are unlikely to be correlated for disparate regions of the ionosphere, variations in the recorded $\mathrm{SNR}_{\mathrm{V}}$ from different GNSS satellites are also unlikely to be correlated from these effects.

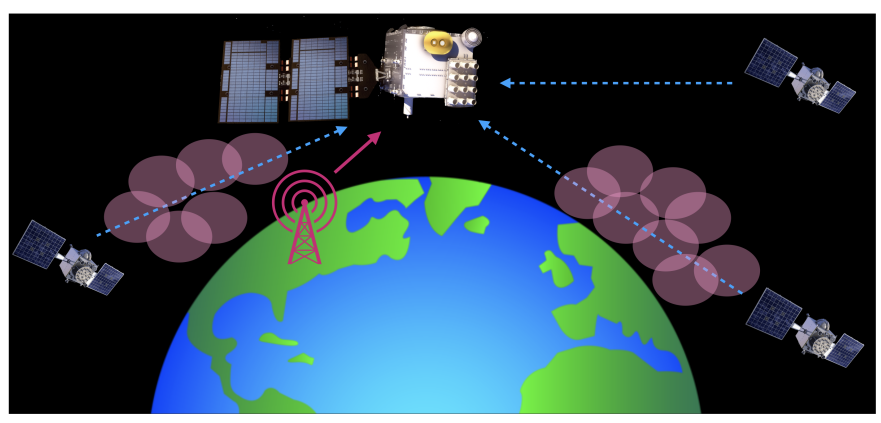

Fig. 2. Observations of ionospheric scintillation from LEO will likely be uncorrelated across tracked transmitters as the plasma variations of signal paths are dissimilar. Conversely, degradation due to RFI source will appear as correlated variations.

The upper panel of Figure 3 shows a typical $\mathrm{SNR}_{\mathrm{V}}$ profile, with amplitude set by the transmitter's power and separation from the receiver, and structure predominately defined by the shape of the receiving antenna gain pattern. Terrestrial RFI 
sources (represented in red in Figure 2) can act to degrade the measured signal strength. For a given antenna, the incident RFI, if in-band, can act to increase the effective noise floor, reducing the $\mathrm{SNR}_{\mathrm{V}}$ on all tracks. Note that this degradation will be correlated across simultaneously tracked signals. As will be discussed, the correlated nature of this degradation is used to detect the presence of RFI. For this section, we will focus on the analysis of GPS data collected by receivers from the COSMIC-2 mission due to their sensitivity and antenna geometry.

\section{A. Effects of RFI on $S_{N} R_{V}$}

The effects of RFI on $\mathrm{SNR}_{\mathrm{V}}$ were clearly observed after the launch of COSMIC-2 in mid 2019 [38]. The impact of RFI is prominent on COSMIC-2 due to the combination of reduced altitude, antenna design/orientation, and a more sensitive RF front-end relative to previous GNSS observing missions. The middle and lower panels of Figure 3 show examples of the effects of terrestrial RFI on the $\mathrm{SNR}_{\mathrm{V}}$ profile (regions in pink), for weak and strong RFI, respectively. Note that while both cases feature oscillations with periodicity on the order of 10-50 seconds, the strong RFI case (lower panel) includes a overall depression in $\mathrm{SNR}_{\mathrm{V}}$ for the duration RFI is present. These strong RFI features vary on a timescale associated with the motion of the spacecraft over a particular region. The specific cause of the higher frequency oscillations is unknown to the authors, but is likely due to transmitter configuration.
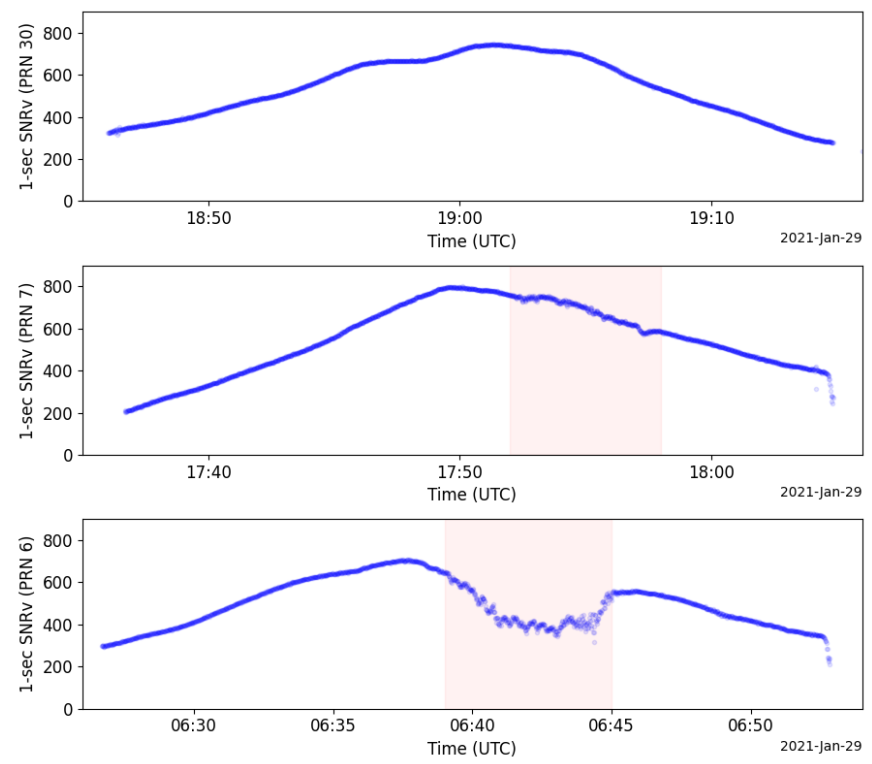

Fig. 3. Top) Typical $\mathrm{SNR}_{\mathrm{V}}$ profile as tracked by the POD antenna of COSMIC-2. Middle) Effects of weak RFI on an $\mathrm{SNR}_{\mathrm{V}}$ profile, RFI duration highlighted in pink. Bottom) Example of strong RFI on $\mathrm{SNR}_{\mathrm{V}}$, showing degradation.

While the strong RFI case appears as a feature distinct from most atmospheric phenomena, the weak case can be easily confused with ionospheric scintillation. This became evident in the accidental triggering of automated processes on the COSMIC-2 receiver that are designed to collect high rate data during scintillation events. As mentioned above, one approach to distinguish these RFI induced oscillations from actual ionospheric scintillation is to check for similar oscillations on simultaneous tracks, especially if it appears on the upward looking antenna tracks. The upper panel of Figure 4 shows $\mathrm{SNR}_{\mathrm{V}}$ of simultaneous tracks during a significant RFI event, displaying the correlation in resulting SNR variations. These GNSS transmitters are well distributed in sky above the receiver, and the signals traverse through regions of the ionospheric plasma from different directions, which are unlikely to have synchronized density irregularities.
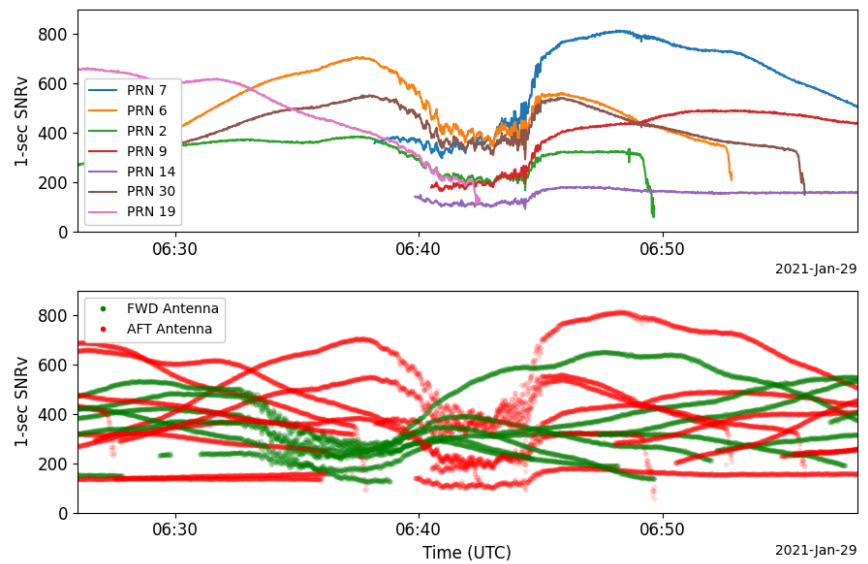

Fig. 4. $\mathrm{SNR}_{\mathrm{V}}$ of simultaneously tracked transmitters showing strong correlation in SNR variations (top), and an antenna dependence and latency, and implicit directionality (bottom).

The ram and anti-ram pointing antennas on COSMIC-2 satellites enable the separable observation of RFI in front and behind the spacecraft. The lower panel of Figure 4 plots $\mathrm{SNR}_{\mathrm{V}}$ from tracks on the ram facing antenna in green, and the anti-ram antenna in red as the spacecraft traverses a region containing a strong RFI source. We observe the presence of the RFI source in front of the spacecraft (on the green traces first), which slowly attenuates then transitions to the rear of the spacecraft (red traces). This correlated but latent appearance of RFI, in combination with knowledge of the antenna orientations, gives directional information about the source of RFI. It should be made clear that this effect does not mean the spacecraft passed directly over the source, but simply that the line of sight to the RFI transmitter passes from one antenna to the next.

Given the strong cross correlation of the effects of RFI on $\mathrm{SNR}_{\mathrm{V}}$ from tracks occurring on the same antenna, detection and quantification of these terrestrial RFI events was pursued via the development of an algorithm that used $\mathrm{SNR}_{\mathrm{V}}$ as the observable.

\section{B. RFI Detection Algorithm}

An RFI detection algorithm was developed based on the assumption that variations between tracks of separate PRNs should be uncorrelated. RFI incident on the antenna will result in the correlated inter-track degradation discussed above, therefore the observation of strong correlation in oscillations of $\mathrm{SNR}_{\mathrm{V}}$ across various tracks is a good indicator of the presence of RFI. 
While the large-scale depression of $\mathrm{SNR}_{\mathrm{V}}$ is a clear effect of strong RFI, the geometric variation of gain pattern effects in $\mathrm{SNR}_{\mathrm{V}}$ occur on comparable timescales. Therefore, they are not trivially separable with simple filtering. Fortunately, the higher frequency signatures of RFI are found to be easily correlated and separable, even for very weak RFI. As such, an algorithm for RFI detection is implemented using a filtered form of the $\mathrm{SNR}_{\mathrm{V}}$. Future work will enable the detection of the slower RFI induced variations as well by using models to predict the received $\mathrm{SNR}_{\mathrm{V}}$, such that comparison to the detected $\mathrm{SNR}_{\mathrm{V}}$ will indicate anomalous increases in the total system noise.

Through experimentation, we found that using a band pass filtered $\mathrm{SNR}_{\mathrm{V}}$ yielded robust detection by removing the lowfrequency geometric effects as well as high frequency, spurious oscillations that could be driven by atmospheric phenomena. A 7th-order digital Butterworth band pass filter with upper and lower corner frequencies of $0.075 \mathrm{~Hz}$ and $0.02 \mathrm{~Hz}$ (respectively) was found to provide satisfactory results. Panel a) of Figure 5 shows $\mathrm{SNR}_{\mathrm{V}}$ from several simultaneous tracks on the same antenna, while the panel directly below shows the band pass filtered form. The correlation between the oscillations in these residual signals is evident.

A subsequent step is to normalize each of the filtered signals by the original unfiltered $\mathrm{SNR}_{\mathrm{V}}$ of the track. This amounts to effectively dividing the traces in panel b) of Figure 5, by their corresponding unfiltered values in panel a). These normalized, filtered values are shown in panel c), demonstrating a remarkable agreement in amplitude and phasing across the various tracks. This normalization approach enables us to decouple the RFI amplitude from the GNSS signal strength.
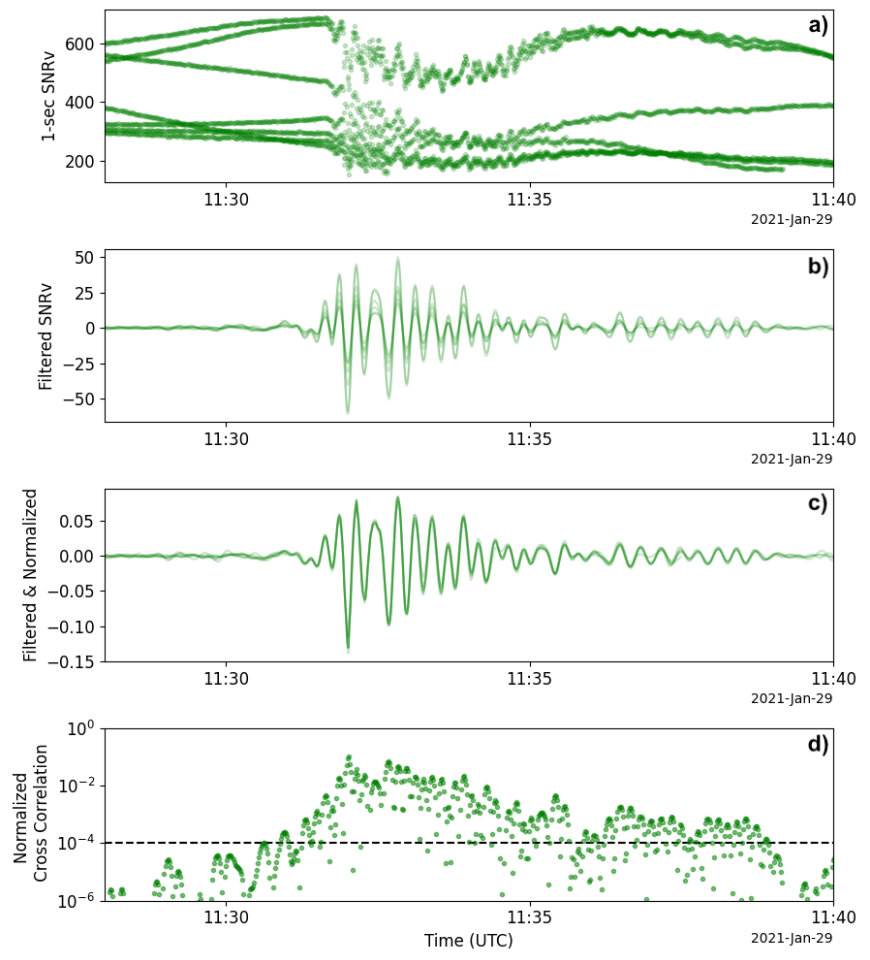

Fig. 5. Example of the algorithmic steps to produce measure of RFI. SNR profiles (a) are bandpass filter (b), then normalized (c), and cross correlated and summed (d).
The final step in the algorithm is to cross-correlate all combinations of the normalized, filtered simultaneous tracks, and sum up the correlation values. From an implementation perspective, this is performed by interpolating all the traces to a contiguous common time-base, where times with no tracking values are zero-filled, allowing for simple cross correlation. The value resulting from this process performed for the traces is shown in panel d) of Figure 5. The duration of RFI incident on a particular antenna can be determined from this metric empirically by selecting a threshold, as indicated by the example horizontal dashed black line in Figure 5. This parameter will be referred to as the "RFI metric" for the remainder of this manuscript.

Note that for a given instant, a particular antenna will provide an RFI metric value using the method just described. This timestamped value is located with the receiver's position information, and oriented by the antenna and spacecraft velocity vector. With this we produce a product that estimates the L-band RFI as viewed from a particular look-direction, at a given location, at a specific time. This high-level product is discussed in detail in the next Section.

\section{Mathematical Representation}

Several representations of the impact of RFI on a GNSS signal have been developed, often which relate back to the carrier-to-noise ratio, $C / N_{0}$ [39]. The SNR output of the TriG is well represented by the convention of Thomas [37], who showed how a 1-bit sampled measurement is related to $\mathrm{SNR}_{\mathrm{V}}$. For convenience, we build a mathematical representation of the RFI metric by working in power $\left(S_{N} R_{P}\right)$ instead of voltage $\left(\mathrm{SNR}_{\mathrm{V}}\right)$ and define the signal power as $S$, the system noise power, $N$, and RFI power, $I$. For a given duration of time, we take $S$ and $N$ as constants, but the RFI as the sum of a constant term and a time-varying term, $I=\bar{I}+\tilde{I}$, where the bar and tilde represent constant and varying, respectively. We define the power signal to noise and interference ratio as,

$$
\mathrm{SNIR}_{\mathrm{P}}=\frac{S}{N+I}=\frac{S}{N+\bar{I}+\tilde{I}}
$$

With this expression we describe the variation in power SNIR which are passed by the filter as,

$$
\begin{aligned}
\sigma^{2}\left(\mathbf{S N I R}_{\mathrm{P}}\right)= & \left(\frac{\partial \mathrm{SNIR}_{\mathrm{P}}}{\partial S}\right)^{2} \sigma^{2}(S) \\
+\left(\frac{\partial \mathrm{SNIR}_{\mathrm{P}}}{\partial N}\right)^{2} \sigma^{2}(N) & \quad+\left(\frac{\partial \mathrm{SNIR}_{\mathrm{P}}}{\partial I}\right)^{2} \sigma^{2}(I) \\
= & \left(\frac{\partial}{\partial I} \frac{S}{N+I}\right)^{2} \sigma^{2}(I)=\frac{S^{2}}{(N+\bar{I})^{4}} \tilde{I}^{2}
\end{aligned}
$$

where we have simply expanded by partial derivatives and the $\sigma$ terms represent the variance of a given variable. The first two terms disappear as they vary on timescale slower than the filter passes $(0.075-0.02 \mathrm{~Hz})$, and the $\bar{I}$ term appears in the denominator as the expanded partial derivative is evaluated at the mean. Assuming the appropriately band pass filter $\mathrm{SNIR}_{\mathrm{P}}$ 
goes like $\sigma\left(\mathbf{S N I R}_{\mathbf{P}}\right)$, we represent the normalized filtered signal by dividing by the total $\mathrm{SNR}_{\mathrm{P}}$ as,

$$
\frac{\mathrm{SNIR}_{\mathrm{P}}}{\mathrm{SNIR}_{\mathrm{P}}}=\frac{\frac{S}{(N+\bar{I})^{2}}}{\frac{S}{N+I}} \tilde{I}=\frac{N+I}{(N+\bar{I})^{2}} \tilde{I}
$$

and arrive at a term nonlinearly related to the variations in RFI power, but completely decoupled from the original signal power. As such, multiple signals experiencing the same system noise and RFI should exhibit an $\mathrm{SNIR}_{\mathrm{P}}$ with this property, regardless of their relative power levels.

The measurements from the receiver are in $\mathrm{SNR}_{\mathrm{V}} \propto$ $\sqrt{\mathrm{SNR}_{\mathrm{P}}}$, so the value shown in panel c) of Figure 5 should go roughly as,

$$
\frac{\mathrm{SNR}_{\mathrm{V}}}{\mathrm{SNR}_{\mathrm{V}}}=\frac{\sqrt{N+I}}{N+\bar{I}} \sqrt{\tilde{I}}
$$

With this, the final step for of the algorithm is the summed cross correlation of the normalized filtered $\mathrm{SNR}_{\mathrm{V}}$ against all other tracks that occur simultaneously. We can sum the crosscorrelation terms across all combinations of $N$ channels to assess the impact of RFI. This metric can be represented as,

$$
M(t)=\sum_{(i, j)}^{N} \sum_{\tau=0}^{T} \frac{\operatorname{SNR}_{\mathrm{V}, \mathrm{i}}(t)}{\operatorname{SNR}_{\mathrm{V}, \mathrm{i}}(t)} \frac{\operatorname{SNR}_{\mathrm{V}, \mathrm{j}}(t-\tau)}{\operatorname{SNR}_{\mathrm{V}, \mathrm{j}}(t-\tau)}
$$

where $N=\left(\begin{array}{l}n \\ 2\end{array}\right)$ represents the number of simultaneous track combinations and $T$ is the total cross correlation window. This expression for $M$ represents the value shown in panel d) of Figure 5.

\section{ObSERVATION OF RFI}

The time-tagged, geolocated, directional metric discussed in the previous section provides a useful measurement of L-band RFI which we can study spatially, temporally, and as a function of amplitude. As detailed in the previous section, it should be noted that this parameter is a measure of RFI amplitude within the digital filter bandwidth $(0.02-0.075 \mathrm{~Hz})$, relative to the total system noise, $N$. This section explores several initial observations, with analytic caveats, from four missions shown in Figure 1, to display the informational content of the metric.

\section{A. Metric Amplitude}

The RFI metric outlined in Section III varies over several orders of magnitude, as is shown in the lower panel of Figure 5, with an amplitude that is set by the RFI source as well as the operating conditions of the receiver. A receiver using an antenna with a line of sight to the source will clearly experience a stronger degradation than one aimed away. Hence, each mission will have an RFI metric distribution that is shaped by the receiving antenna gain pattern, filtering of the RF front-end, and range to the source.

The upper panel of Figure 6 shows the amplitude distributions for the four missions, using all available samples. Immediately apparent is the distribution pairing of the two COSMIC
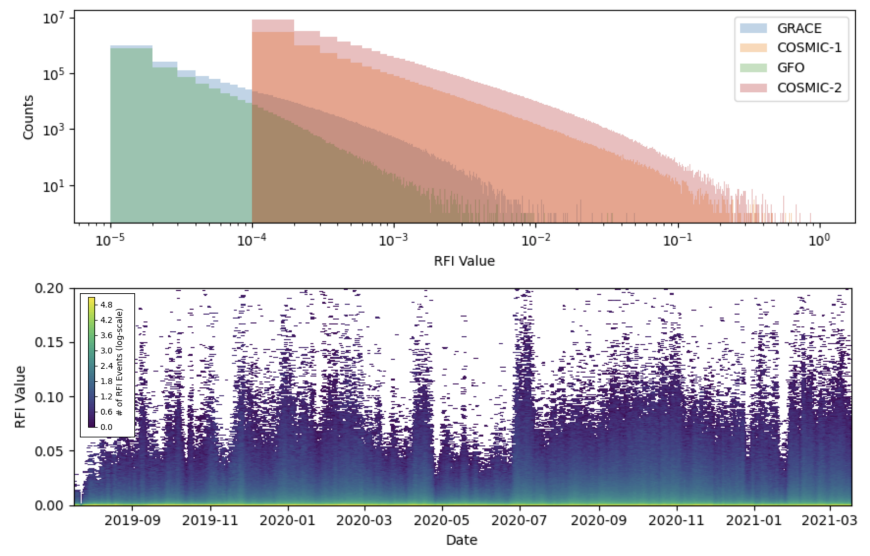

Fig. 6. Full mission RFI metric amplitude distributions (top) shows the range of this parameter over several orders of magnitude, as well as the relation between missions. Variation of the global RFI distribution (bottom) is shown for COSMIC-2, displaying temporally varying RFI.

missions (orange and red) and the two GRACE missions (blue and green), driven predominantly by the orientation of the antennas. The GRACE missions are less affected by RFI due to their zenith-facing antennas than the COSMIC missions that have antennas facing the horizon. The COSMIC missions have both low-gain POD antennas and highly-directional antennas with gain directed toward the earth's limb. This study uses the POD antenna data, but future work will use the more sensitive data from the limb antennas. The lower limits of all four distributions are set by an arbitrarily determined threshold to eliminate samples taken without the clear presence of RFI. Also note that the "areas under the curve" differ between the missions with only two satellites (GRACE/GFO) verses those with 6 (COSMIC/COSMIC-2).

In all cases, these distributions easily range over 3-4 orders of magnitude. The extremely high amplitude tails of these distributions are commonly associated with receiver software or hardware issues that cause common variation on all channels. The very low end of the distributions are likely to contain little, if any, RFI. The intervening range contains values that appear to be driven by terrestrial transmitters. Note that these wide ranges of values make plotting the RFI metric challenging, especially when comparing measurements from different missions. In the following discussion, RFI events will be aggregated and compared either by the amplitude of the RFI metric, or simply by counting "events" as defined by the metric crossing a threshold. The specifics will be made explicit in each case.

We also use these amplitude distributions to gain insight into the temporal variation of the global state of RFI. The lower panel of Figure 6 shows the time variation of the amplitude distribution for COSMIC-2 receivers using histograms produced with a sliding two month window. The number of counts are adjusted for satellite outages, and significant features in the plot are uncorrelated with events such as software updates. Rapid changes to the distribution, such as around July of 2020, indicate the onset of RFI activity somewhere on the planet. 

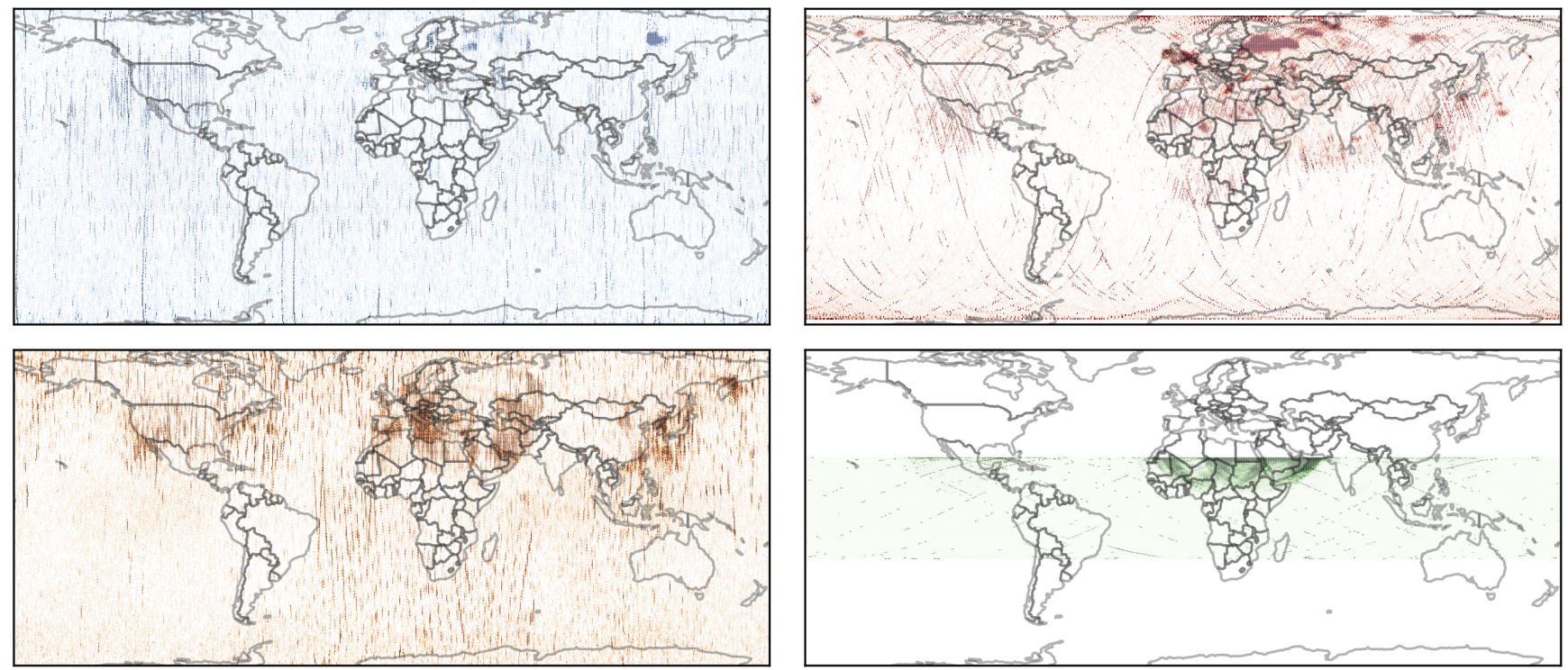

Fig. 7. Full mission geographic distribution of RFI, from GRACE (upper left, threshold=0.001), and GFO (lower left, threshold=0.0005), COSMIC (upper right, threshold=0.05) and COSMIC-2 (lower right, threshold=5). RFI values are integrated over the mission duration into $36 \mathrm{~km}$ grid cells, and plots are thresholded to highlight dominant features.

\section{B. Geographic Distribution}

We visualize the global distribution of RFI sources by binning the surface of the planet into $36 \mathrm{~km}$ EASE grid cells [40], then integrating measurements that occur over a given bin for a duration of time. Figure 7 shows the binning/integration of the RFI metric value for each mission, integrated for the full mission duration (or up to the end of 2020 for operational missions), acting as a summary of the nature of RFI for each mission. The color in each plot has been thresholded to a value (see caption), which is set by the mission duration and sensitivity of the antenna/receiver. Regions with particularly strong, frequent RFI are visible as solid patches, while scattered detections are associated with isolated events or receiver software/hardware issues. The high inclination orbits of GRACE, GFO, and COSMIC provide near complete global coverage, while COSMIC-2 is limited to $\pm 24^{\circ}$. This limited coverage enables a more frequent revisit rate for these regions, enabling higher spatiotemporal resolution of the activity there.

The "full mission" plots of Figure 7, while useful as an overview, do not convey the temporal variation of RFI shown in the bottom of Figure 6. Interesting events and dynamics are made visible by limiting the integration window of these plots to specific durations of time. Figure 8 shows the RFI as measured by COSMIC integrated over yearly windows for a decade-long span. RFI activity is shown to vary significantly on this time scale, but can change as rapidly as monthly or even daily for certain events. Inherently the trade off between coverage and revisit rate limits the resolution of a given event, though this trade can be mitigated through the use of a sliding integration window. The Supplemental Material includes videos with 1 month to 6 month sliding windows that indicate the onset and duration of particular events.

It should be noted that the locations highlighted on the maps

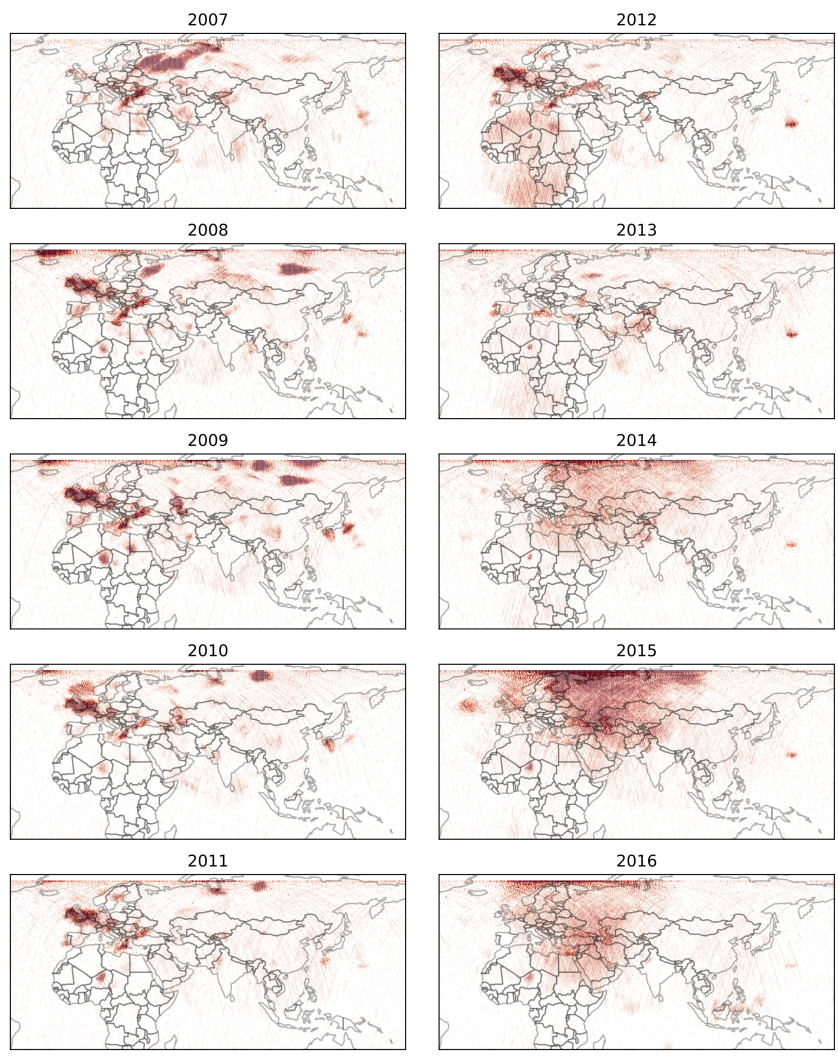

Fig. 8. One-year integrated values from COSMIC showing the temporal variation of RFI over Europe, Asia, and Africa. Effects of the Russia-Ukraine conflict visible from 2014-2016. 
in this article indicate the position of the receiver, not the source of RFI. Knowledge of the antenna orientation provides some insight into the relative direction of that RFI source. An example of this is shown in Figure 9, where the counts of RFI events (not the metric amplitudes) from the antiram-looking antennas have been subtracted from the ram-looking counts, providing improved localization of the source. In this figure, blue indicates an RFI source in front of the spacecraft, while red is behind the spacecraft. Neutral regions are where the relative number of events are roughly equal. These directional measurements are only crude indicators of the source location, and additional analysis using the spacecraft velocity vector, gain patterns, and inter-satellite comparison is needed to better assess directionality and enhance the location resolution of RFI sources.

Even though GRACE and GFO have omnidirectional, zenith pointing antennas, the RFI they detect is similarly displaced from the spacecraft location. The shape of the spacecraft below the antenna limits the observable region, and actually makes detection to the sides of the spacecraft more sensitive. This is observable in shape of the detection pattern of GFO over Western Russia/Syria as strong side lobes relative to the direction of spacecraft motion (nearly latitudinal). As such, the detection pattern falls roughly above a known jammer/spoofer located in Syria [6], [12].

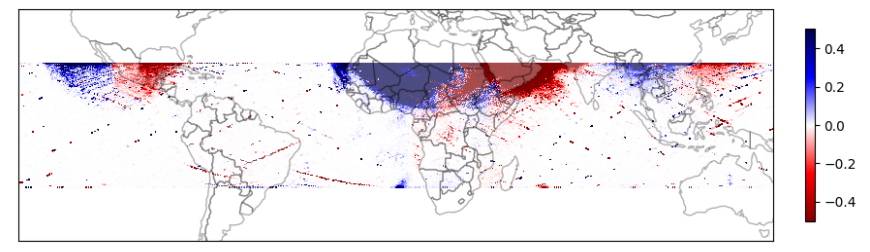

Fig. 9. Representation of RFI directionality produced by subtracting number of RFI events detected in front (blue) verses behind (red) COSMIC-2 satellites from 2020 .

Inter-mission analysis helps yield a fuller understanding of these sources. For example, Figure 10, displaying integrated detection events from GFO and COSMIC-2 (on different scales), shows that by combining the coverage and resolution between different missions, we are better able to see that some of the RFI detected by COSMIC-2 over Africa is likely due to the same Syrian transmitter. This was not obvious from inspection of the COSMIC-2 data in isolation. Other locations, such as Libya/Tunisia and North Korea appear as potential sources in Figure 10. Furthermore, the up/down directionality of the RFI can be assessed through comparison of zenith pointing detection to those made on other spacecraft similar times with ground-viewing antenna. By this approach, it was easily (and unsurprisingly) determined that all observed RFI sources were, in fact, terrestrial.

Finally, analysis of multiple missions creates a 20 year record of terrestrial RFI, captured in the plots of Figure 7, or even better the inter-mission compilation video included as Supplemental Material. GRACE, while the least sensitive of the missions, stretches this record back to 2002, giving

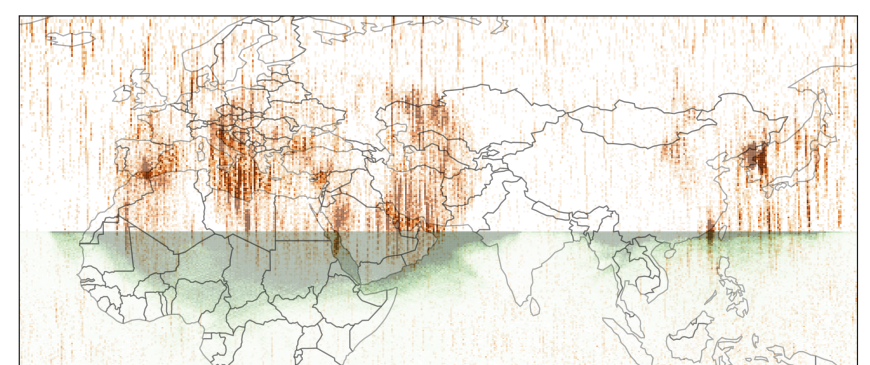

Fig. 10. Overlay of the detections from GFO (orange), and COSMIC-2 (green, latitudes below $24^{\circ}$ ) from 2020. The features of both outline a known RFI source in Syria, as well as another potential sources near Libya/Tunisia and North Korea.

a solitary perspective on certain events, such as interesting activity over North-East Russia.

\section{False RFI Detection Examples}

While the measurements above show clear indications of terrestrial RFI sources, there is also contamination from a variety of artifacts which produce correlated signals across the tracks of a receiver. These artifacts are highly mission specific, deriving from issues with receiver hardware and/or software, as well as possible environmental effects. In general, the slight colored haze that appears on all four plots of Figure 7 is due to random, uncommon false RFI detections distributed over the map.

The scattered blue lines on the GRACE plot of Figure 7 occur at roughly constant latitude (more easily visible in GRACE video provided in Supplemental Material), the cause of which is currently unknown to the authors. Also on GRACE, there was also known cross coupling between the POD antenna and RO antenna, which caused strong, but sporadic, detections. GFO exhibits a similar looking, but likely different effect to GRACE. Here, RFI detections appear to precess longitudinally (again, more visible in videos contained in the Supplemental Material) for a yet to be determined reason. GFO also suffered from an issue with repeated timetagged data (of different values), creating correlated ringing in the filtered outputs, an effect often associated with tracks from unhealthy transmitters.

COSMIC demonstrated several forms of synchronized artificial disturbances, including instantaneous drops in SNRv across channels, as well as correlated noise likely due to a known hardware temperature-related issue. These phenomena produced intense, continuous detections, observed as the dark arcs over the ocean in the COSMIC plot of Figure 7, as well as small, scattered events. COSMIC-2, in contrast, exhibits the least artificial RFI. Prior to 2020, there was a software issue resulting in a reduced sample rate for the SNRv which produces weak, spurious detections uniformly. Since resolving this issue, COSMIC-2 produced RFI detections consistently over known interference regions, with few to none over places like the Southern Pacific. 


\section{Correlations of RFI with Notable Events and Locations}

In the above discussion we have shown an ability to associated RFI with specific regions, and even observed dynamics over those regions. The next reasonable question is - what could be driving these events? Through the inspection of animations of global RFI (see Supplemental Materials), similar to those shown in Figure 8, notable RFI activity can be correlated with known geopolitical events. It is beyond the scope of this work to make inferences on how these events result in the activation of L-band transmitters, but the correlation is clear.

A marked example is visible in the 2012 panel of Figure 8 as a circular spot centered over Nigeria. This event, roughly one month in duration, correlates well with a state of emergency declared in Nigeria due to attacks from the militant group, Boko Haram. The extent and circularity of the detections actually drove the authors to consider the possibility that the RFI was from an orbital platform, but a comparison between measurements made on the zenith-pointing antennas of GRACE to the horizon pointing antennas of COSMIC easily determine this RFI to be terrestrial in origin.

Also visible in the 2015 panel of Figure 8 is activity over Western Russian, roughly correlated with escalating tensions between Russia and Ukraine over the annexation of the Crimean peninsula. A similar coverage of RFI activity is present in 2014 and and 2016 (roughly the duration of these tensions), but is almost entirely gone by the end of the COSMIC mission in 2018. Other transient activity is visible in the animations and panels of Figure 8 that have yet to be correlated with particular events or even known locations of significance. From these example, it can be seen that this new dataset from the last two decades provides a unique perspective on the planet.

\section{Conclusions}

An array of scientific investigations rely on GNSS constellations to produce the "signals of opportunity" which allow for a unique, low cost method to measure the Earth's atmosphere and many surface parameters. Unfortunately, these weak signals are vulnerable to interference, often produced for military purposes. Achieving an understanding of the global RFI environment is vital to improving the quality of scientific measurements determined from GNSS receivers.

The algorithm discussed in this work has demonstrated an ability to sensitively detect RFI using variations in the measured signal strength in a manner distinguishable from other ionospheric effects of a similar form. Using the time and position determined by the GNSS receiver, we have shown that these detections can be used to estimate the locations of RFI transmitters, intentional or otherwise, as well as bulk changes in the regional RFI environment. This information is immediately applicable as a quality flag for the associated science measurements, but is useful for planning future instruments and missions that may be affected by these terrestrial transmitters. New sources of L-band RFI, such as the Ligado network, will be coming online as soon as late 2021 and having a global estimate of the impact of these sources on signal quality is critical.
Furthermore, a determination of the nature of the RFI source as intentional or unintentional can likely be characterized through the amplitude and dynamics of signal degradation. A better understanding of the relation of these activities to known events could be valuable to interpret the intentions of global actors. For example, does the onset of RFI lead or lag the onset of a known conflict or event? A 20 year record of these events could prove useful for historical context as well.

The work outlined here was limited in scope to L1 CA GPS signals collected on the the POD antennas, focusing primarily on detection alone. Future work will investigate the impact of RFI on other signals with different frequencies collected by the same instruments, providing a better understanding of the nature of the RFI. Extending the detection capability to slowlyvarying signal amplitudes will indicate presence of some troublesome high-power RFI sources. Analysis of RFI using the beam-steerable RO antennas of COSMIC- 2 has the potential to enhance azimuthal resolution of the source location, as well as provide additional context to the impact on atmospheric measurements. Spectral analysis of the RFI induced variations to $\mathrm{SNR}_{\mathrm{V}}$ could allow for distinguishing between RFI sources that differ in nature. Performing similar analyses to additional past and future missions carrying TriG/Blackjack receivers will also occur, further extending the coverage and resolution of these RFI measurements.

This task described in this paper was performed at the Jet Propulsion Laboratory, California Institute of Technology, under a contract with the National Aeronautics and Space Administration with funding from the Space and Missile Systems Center (SMC), United States Space Force. The authors would like to thank colleagues at other institutions for valuable contributions to this work. Exchanges with Doug Hunt at UCAR helped explain issues observed with COSMIC data. Conversations with Captain Peter Nork provided valuable context to the effects of GPS outages on commercial and private aviation. (C) 2021. California Institute of Technology. Government sponsorship acknowledged.

\section{REFERENCES}

[1] T. P. Yunck, S. C. Wu, J. W. I. T. on, and 1990, "Precise tracking of remote sensing satellites with the Global Positioning System," ieeexplore.ieee.org. [Online]. Available: https://ieeexplore.ieee.org/abstract/document/45753/

[2] Z. Kang, B. Tapley, S. Bettadpur, J. Ries, P. Nagel, and R. Pastor, "Precise orbit determination for the GRACE mission using only GPS data," Journal of Geodesy, vol. 80, no. 6, pp. 322-331, Sep. 2006. [Online]. Available: https://link.springer.com/article/10.1007/s00190006-0073-5

[3] J. A. Volpe, "VULNERABILITY ASSESSMENT OF THE TRANSPORTATION INFRASTRUCTURE RELYING ON THE GLOBAL POSITIONING SYSTEM," National Transportation Systems Center.

[4] A. P. Cerruti, P. M. Kintner, D. E. Gary, A. J. Mannucci, R. F. Meyer, P. Doherty, and A. J. Coster, "Effect of intense December 2006 solar radio bursts on GPS receivers," Space Weather, vol. 6, no. 10, Oct. 2008. [Online]. Available: https://agupubs.onlinelibrary.wiley.com/doi/full/10.1029/2007SW000375

[5] E. Yizengaw and K. M. Groves, "Longitudinal and seasonal variability of equatorial ionospheric irregularities and electrodynamics," Space Weather, vol. 16, no. 8, pp. 946-968, 2018. [Online]. Available: https://agupubs.onlinelibrary.wiley.com/doi/abs/10.1029/2018SW001980

[6] "Exposing GPS Spoofing in Russia and Syria," Tech. Rep., Mar. 2019.

[7] M. L. Psiaki, T. E. Humphreys, and B. Stauffer, "Attackers can spoof navigation signals without our knowledge. Here's how to fight back GPS lies," pp. 1-9, Jul. 2016. 
[8] A. M. Wolff, D. M. Akos, and S. Lo, "Potential radio frequency interference with the GPS L5 band for radio occultation measurements," Atmospheric Measurement Techniques, vol. 7, no. 11, pp. 3801-3811, 2014. [Online]. Available: https://amt.copernicus.org/articles/7/3801/2014/

[9] K. Wesson and T. Humphreys, "Fleets of unmanned aircraft may soon scan terrain for forest fires and deliver FedEx packages. Yet drones' security flaws allow them to be readily hijacked with simple technologies," Nature Publishing Group, vol. 309, no. 5, pp. 54-59, Nov. 2013. [Online]. Available: http://dx.doi.org/10.1038/scientificamerican1113-54

[10] M. Bonnedal, J. Christensen, A. Carlström, and A. Berg, "Metop-GRAS in-orbit instrument performance," GPS Solutions, vol. 14, no. 1, pp. 109-120, Sep. 2009. [Online]. Available: http://link.springer.com/10.1007/s10291-009-0142-3

[11] O. Isoz, S. A. Buehler, K. Kinch, M. Bonnedal, and D. M. Akos, "Interference from terrestrial sources and its impact on the GRAS GPS radio occultation receiver," Radio Science, vol. 49, no. 1, pp. 1-6, Jan. 2014. [Online]. Available: https://agupubs.onlinelibrary.wiley.com/doi/full/10.1002/2013RS005243

[12] M. J. Murrian, L. Narula, P. A. Iannucci, S. Budzien, B. W. O'Hanlon, and T. E. Humphreys, "GNSS Interference Monitoring from Low Earth Orbit," arXiv, Sep. 2020. [Online]. Available: https://arxiv.org/abs/2009.04093v1

[13] Z. Liu, S. Lo, and T. Walter, "Characterization of ADS-B Performance under GNSS Interference," Proceedings of the rd International Technical Meeting of the Satellite Division of The Institute of Navigation, pp. 3581-3591, Sep. 2020. [Online]. Available: http://www.ion.org/publications/abstract.cfm?jp=p\&articleID=17675

[14] D. Entekhabi, S. Yueh, P. O'Neill, K. Kellogg, and 2014, "SMAP Handbook." [Online]. Available: http://scholar.google.comjavascript:void(0)

[15] E. Yizengaw, M. B. Moldwin, D. Galvan, B. I. J. o Atmospheric, and 2008, "Global plasmaspheric TEC and its relative contribution to GPS TEC," Elsevier. [Online]. Available: https://www.sciencedirect.com/science/article/pii/S1364682608001193

[16] G. A. Hajj, "A technical description of atmospheric sounding by GPS occultation," pp. 1-19, Apr. 2002.

[17] R. A. Anthes, P. A. Bernhardt, Y. C. B. o the, and 2008, "The COSMIC/FORMOSAT-3 mission: Early results," journals.ametsoc.org. [Online]. Available: https://journals.ametsoc.org/view/journals/bams/89/3/bams-89-3313.xml

[18] W. S. Schreiner, J. P. Weiss, R. A. Anthes, J. Braun, V. Chu, J. Fong, D. Hunt, Y. H. Kuo, T. Meehan, W. Serafino, J. Sjoberg, S. Sokolovskiy, E. Talaat, T. K. Wee, and Z. Zeng, "COSMIC-2 Radio Occultation Constellation: First Results," Geophysical Research Letters, vol. 47, no. 4, p. e2019GL086841, Feb. 2020. [Online]. Available: https://agupubs.onlinelibrary.wiley.com/doi/full/10.1029/2019GL086841

[19] M. P. Clarizia, C. P. Gommenginger, S. T. Gleason, M. A. Srokosz, C. Galdi, and M. Di Bisceglie, "Analysis of GNSS-R delay-Doppler maps from the UK-DMC satellite over the ocean," Geophysical Research Letters, vol. 36, no. 2, pp. n/a-n/a, Jan. 2009. [Online]. Available: http://doi.wiley.com/10.1029/2008GL036292

[20] V. U. Zavorotny, S. Gleason, E. Cardellach, and A. Camps, "Tutorial on Remote Sensing Using GNSS Bistatic Radar of Opportunity," IEEE Geoscience and Remote Sensing Magazine, vol. 2, no. 4, pp. 8-45. [Online]. Available: http://ieeexplore.ieee.org/document/6985926/

[21] C. Chew and E. Small, "Description of the UCAR/CU Soil Moisture Product," Remote Sensing, vol. 12, no. 10, pp. 1558-26, May 2020. [Online]. Available: https://www.mdpi.com/2072-4292/12/10/1558

[22] C. Ruf, P. S. Chang, M. P. Clarizia, S. Gleason, and Z. Jelenak, "CyGNSS handbook. Cyclone global navigation satellite systems," 2018. [Online]. Available: http://scholar.google.comjavascript:void(0)

[23] T. A. Herring, T. I. Melbourne, M. H. Murray, M. A. Floyd, W. M Szeliga, R. W. King, D. A. Phillips, C. M. Puskas, M. Santillan, and L. Wang, "Plate Boundary Observatory and related networks: GPS data analysis methods and geodetic products," Reviews of Geophysics, vol. 54, no. 4, pp. 759-808, Dec. 2016. [Online]. Available: https://agupubs.onlinelibrary.wiley.com/doi/full/10.1002/2016RG000529

[24] Y. Bock, D. Melgar, B. C. B. o. the, and 2011, "Realtime strong-motion broadband displacements from collocated GPS and accelerometers," pubs.geoscienceworld.org. [Online]. Available: https://pubs.geoscienceworld.org/bssa/article-lookup/101/6/2904

[25] J. Y. Liu, Y. J. Chuo, S. J. Shan, Y. B. Tsai, Y. I. Chen, S. A. Pulinets, and S. B. Yu, "Pre-earthquake ionospheric anomalies registered by continuous GPS TEC measurements," Annales Geophysicae, vol. 22, no. 5, pp. 1585-1593, Apr. 2004. [Online]. Available: https://angeo.copernicus.org/articles/22/1585/2004/

[26] R. M. Allen and A. Ziv, "Application of real-time GPS to earthquake early warning," Geophysical Research Letters, vol. 38, no. 16, Aug. 2011.

[27] D. N. Dong and Y. Bock, "Global Positioning System Network analysis with phase ambiguity resolution applied to crustal deformation studies in California," Journal of Geophysical Research: Solid Earth, vol. 94, no. B4, pp. 3949-3966, Apr. 1989. [Online]. Available: https://agupubs.onlinelibrary.wiley.com/doi/full/10.1029/JB094iB04p03949

[28] B. D. Tapley, S. Bettadpur, M. Watkins, and C. Reigber, "The gravity recovery and climate experiment: Mission overview and early results," Geophysical Research Letters, vol. 31, no. 9, May 2004. [Online]. Available: https://agupubs.onlinelibrary.wiley.com/doi/full/10.1029/2004GL019920

[29] R. P. Kornfeld, B. W. Arnold, M. A. Gross, N. D. J. o. spacecraft, and 2019, "GRACE-FO: the gravity recovery and climate experiment follow-on mission," arc.aiaa.org. [Online]. Available: https://arc.aiaa.org/doi/abs/10.2514/1.A34326

[30] O. B. Andersen, S. S. Geophysical, and 2005, "GRACE-derived terrestrial water storage depletion associated with the 2003 European heat wave," Wiley Online Library. [Online]. Available: https://agupubs.onlinelibrary.wiley.com/doi/abs/10.1029/2005GL023574

[31] B. F. Zaitchik, M. R. J. of, and 2008, "Assimilation of GRACE terrestrial water storage data into a land surface model: Results for the Mississippi River basin," journals.ametsoc.org.

[32] B. D. Tapley, M. M. Watkins, F. Flechtner, C. R. N. climate, and 2019, "Contributions of GRACE to understanding climate change," nature.com. [Online]. Available: https://www.nature.com/articles/s41558019-0456-2

[33] J. B. Thomas, "Functional description of signal processing in the Rogue GPS receiver," 1988. [Online]. Available: https://core.ac.uk/download/pdf/42830809.pdf

[34] R. Haddad, K. Kovach, R. S. . I. I. Position, and 2020, "GPS Modernization and Beyond," ieeexplore.ieee.org. [Online]. Available: https://ieeexplore.ieee.org/abstract/document/9110167/

[35] L. T. W. Group, "LightSquared Technical Working Group Report," vol. 11-109, 12-340. [Online]. Available: https://www.fcc.gov/document/fccapproves-ligado-1-band-application-facilitate- $5 \mathrm{~g}$-iot

[36] J. Bhatti and T. E. Humphreys, "Hostile Control of Ships via False GPS Signals: Demonstration and Detection," NAVIGATION, Journal of the Institute of Navigation, vol. 64, no. 1, pp. 51-66, Mar. 2017.

[37] J. B. Thomas, "Signal-Processing Theory for the TurboRogue Receiver," JPL Publication, pp. 1-95, Apr. 1995.

[38] P. Straus and J. Braun, Technical discussions between JPL, Aerospace, and UCAR, Oct 2018.

[39] J. W. Betz, "Effect of Partial-Band Interference on Receiver Estimation of C/N0: Theory," The MITRE Corporation, pp. 817-828, Jan. 2001. [Online]. Available: http://www.ion.org/publications/abstract.cfm?.jp=p\&articleID=195

[40] M. J. Brodzik, B. Billingsley, T. Haran, B. Raup, and M. H. Savoie, "EASE-Grid 2.0: Incremental but Significant Improvements for Earth-Gridded Data Sets," ISPRS International Journal of GeoInformation, vol. 1, no. 1, pp. 32-45, Jun. 2012. [Online]. Available: http://www.mdpi.com/2220-9964/1/1/32 